UC-15

Reporting Date: July 1974

Issued: August 1974

\title{
Assay of the Uranium Content of Rover Scrap with the Random Source Interrogation System
}

\author{
by \\ J.E. Foley \\ L.R. Cowder
}


This report was prepared as an account of work sponsored by the United States Government. Neither the United States nor the United States Atomic Energy Commission, nor any of their emplovees, nor any of their contractors, subcontractors, or their employees, makes any warranty, express or implied, or assumes any legal liability or responsibility for the accurasy, completeness or usefulness of any information, apparatus, product or process disclosed, or represents that its use would not infringe privately owned rights.

In the interest of prompt distribution, this LAMS report was not edited by the Technical Information staff.

Printed in the United States of America. Available from

National Technical Information Service

U.S. Department of Commerce

5285 Port Royal Road

Springfield, Virginia 22151

Price: Printed Copy $\$ 4.00$ Microfiche $\$ 1.45$ 


\title{
ASSAY OF THE URANIUM CONTENT OF ROVER SCRAP
}

WTH THE RANDOM SOURCE INTERROGATION SYSTEM

by

J. E. Foley and L. R. Cowder

\begin{abstract}
$\Lambda$ neutron interrogation instrument has been built that determines the uranium content of 10 -liter containers of scrap material from the Rover nuclear rocket program. The instrument determines uranium content by producing fissions in the sample, and then detecting these fissioris by coincidence counting the prompt-fission neutrons and gamma rays. A measurement procedure has been developed that gives assays that are relatively independent of the matrix material in the sample. A normalization procedure has also been developed that eliminates the effect 3 of instrumentation drifts.
\end{abstract}

\section{INTRODUCTION}

The termination of the Rover nuclear rocket program in June 1973 made it necessary to do a complete assay of the entire Rover uranium fuel inventory. This inventory consisted of intact fuel elements, damaged fuel elements, and scrap from the fuel-manufacturing process that had accumulated over the many years of production. The uraniurn content of the intact fuel elements was well known because of the quality coitrol on the production prccess, but the uranium content of the damaged elements and the scrap was nct well known, The uranium in the damaged elements and iu the scrap was to be recovered by the uranium recovery facility at the Los Alamos Scientific Laboratory. Uranium recovery had been going on for many years, but a large backlog of scrap containers still existed at the time of the termination of the Rover program. The damaged elements were broken into small pieces and stored in 10-liter stainless steel "hospital cans" (208-mm-o, d. by 254-mm-high). The scrap, composed of graphite cust and small pieces of fuel eiements, was also stored in these containers.

It was necessary io develop an assay instrument that could be used at the recovery facility to determine the uranium content of the incuming Rover samples, and that could be operated by the personnel at the facility.

For many years, Rover scrap samples had been assayed by the delayed-neutron technique. ${ }^{1}$ This technique, which requires the use of either a Van de Graaff accelerator or a 14-MeV neutron generator, has been shown to be very accurate; but, berause of the complexity of the instrumentation, is not easily used for routine in-plant assay. The gamma-ray assay technique, ${ }^{2}$ which is used for the assay of low-density samples, could 
not be used because these samples, which typically contain 500 to $3000 \mathrm{~g}$ of uranium, were too dense to obtain proper gamma-ray transmission corrections. The nondestructive assay instrument that could possibly do the job was the Random Source Interrogation System ${ }^{3}$ (which is more commonly called the "Random Driver"). This assay system had been used successfully at the Oak Ridge Y-12 plant ${ }^{4}$ for the assay of high-enxiched uranium scrap in small containers (125-mm-o, d. ). The Random Driver, along with other instruments of the same generic type, ${ }^{*}$ determines the ${ }^{235} U$ content of a sample by detecting neutron and gamma rays from fissions which are produced by neutron interrogation from a small neutron source $\left(\sim 10^{6} \mathrm{n} / \mathrm{s}\right)$. Since the enrichment of the uranium in the Rover material was constant $(93 \%)$, measurement of the ${ }^{235} \mathrm{U}$ content, as done by the Random Driver, would be sufficient for the determination of total uranium content.

A Random Driver assay instrument was designed and built especially for the Rover scrap assay problem. A measurement procedure has been developed that results in assays which are relatively independent of the composition of the sample. A normalization technique has been included in this procedure which eliminates the effects of instrumentation drifts.

\section{THEORY OF OPERATION}

A top view of the Random Driver is shown in

Fig. 1. A small fraction of the neutrons from the AmLi source $\left(\sim 10^{6} \mathrm{n} / \mathrm{s}\right)$ interacts with the uranium in the sample, inducing fissions in the ${ }^{235} \mathrm{U}$ atoms. Reiatively few fissions occur in the ${ }^{238} \mathrm{U}$ because the neutron-energy spectrum of the AmLi source is subthreshold ( $1 \mathrm{MeV}$ ) for ${ }^{238} \mathrm{U}$. About two and one-half neutrons and about six gamma rays are

\footnotetext{
"The Isotopic Source Assay Systems manufactured by Intelcom Rad Tech, San Diego, CA; the Random Driver manufactured by National Nuclear Corp. , Redwood City, CA; and FEFI manufactured by Texas Nuclear Corp., Austin, TX.
}

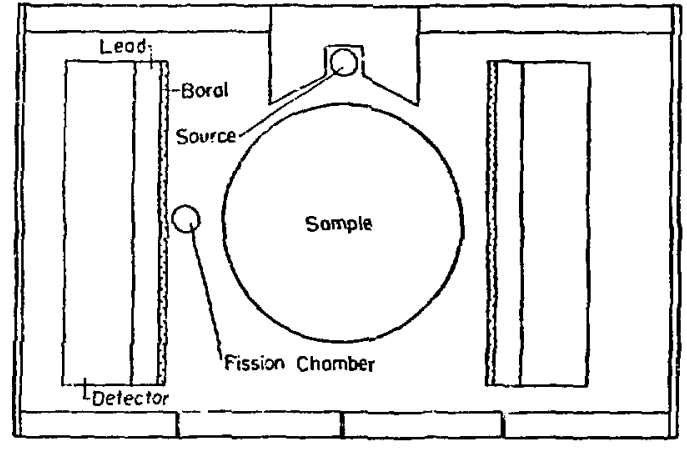

Fig. 1. Top view of the Random Driver. produced when a ${ }^{235} \mathrm{U}$ atom fissions. These neutrons and gamma rays are detected, in the presence of the relatively intense neutron flux from th: AmLi source, by coincidence counting with two fast plastic scintillation detectors located on opposite sides of the sample. The fission neutrons and gamma rays are time-correlated (produced two or more at a timie), whereas the AmLi source neutrons are produced randomly (uncorrelated, produced one at a time). The coincidence-counting technique. which requires the detection of two neutrons or gamma rays within a very short period of time, is used to distinguish the time-correlated, inducedfission neutrons and gamma rays from the uncorrelated, or random, source neutrons. if a neutron or gamma-ray detection in one of the fast plastic siintillation detectors is followed closely ( $<40 \mathrm{~ns})$ by a detection in the seccnd detector, then a coincidence event has been observed. The probability that such an event occurs is high for the timecorrelated neutrons and gamma rays from fission, and it is low for the uncorrelated neutrons from the random source. The coincidence rate, being proportiona! to the quantity of the ${ }^{235} U$ in the sample. therefore frovides a "signature" for determining the uranium content of the sample.

The energy spectrum of the interrogating neutrons from the source is kept "hard" (high energy) in order to obtain good penetration into the sample. A thermalized-neutron source will 
increase the induced-fission rate by a factor of at Icast 10, but assays made with this type of source are very sensitive to the density of the usanium and the ratrix material in the sample ${ }^{5}$ because of the poor neutron penetration into the sample. Hardspectrum interrogation is justified, in spite of the lower inducea-fission rate, because the assays, though not as precise, are ccrisistently more accurate than those made by thermal-neutron interrogation.

Three corrections must be made to the measured coincidence rate before an accurate assay can be given. Corrections must be made for (1) a background change due to the presence of the sample in the counter, (2) neutron thermalization within samples containing hydrogenous materials, and (3) attenuation of the fission neutrons and gamma rays within the sample.

A. Background Correction

A background coincidence rate of 12,0 counts/s is observed in tho Random Driver designed for the assay of the Rover scrap samples. Most of this background is from cosmic rays that, in passing through the system, produce simultaneous counts in both detectors, resulting in a coincidence count. A lower background rate is observed when the sample is placed into the counter, because some of the cosmic rays that ordinarily would travel through both detectors, and thus produce coincidence count $:$, are stopped within the sample; i, e., the sample shields the second detector from a fraction of the cosmic rays. The background coincidence rate with the sample in the counter cannot be measured in the presence of the AmLi source because this background coincidence rate cannot be distinguished from the induced-fission rate. Since it would be very time-consuming to remove the source and to measure the background for each sample, a different approach is necessary. It was determined experimentally that the change in the background due to the sample is a function of only the mass of the sample and not a function of the composition of the sample for the low- and intermediate- $Z$ materials investigated. Figure 2 illustrates this for the Rover scrap container filled with various densities of materials that are expected to be found in the samples: metal, graphite, and graphite flour. The background rate can be calculated from the weight of the sample. A separate background measurement thus does noi need to be made for each sample.

\section{B. Fission-Chamber Correction}

Samples containing hydrogenous matrix materials show higher induced responses per gram of 235 U than do samples without such materials. A $235 \mathrm{U}$ fission chamber, positioned inside the Random Driver close to the sample, is used to monitor the thermal flux within the sample. An increase in the response of the fission chamber indicates that there is a similar increase in the response from the sample itself. Normalization of the induced coincidence rate of the sample to the count rate of the fission chamier corrects the assay by eliminating the thermalization effects of t'.1e hydrogen. ${ }^{6}$

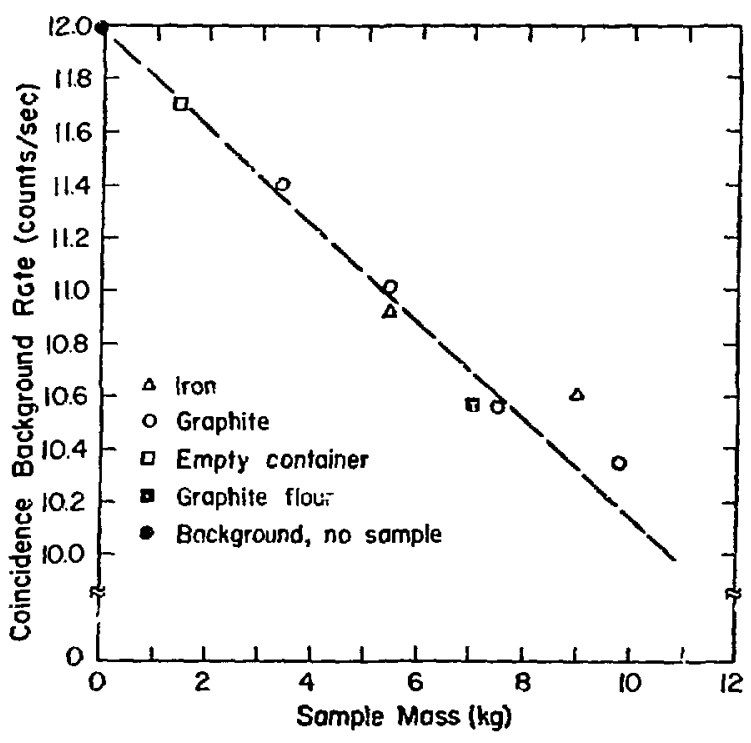

Fig. 2. Background coincidence rate of the Random Driver for various sample compositions and masses. 


\section{Attenuation Correction}

A fraction of the fission neutrons and gammo rays that are produced within the sample from the neutron interrogation are captured by the material inside the sample. In addition, elastic scattering of 2 fission neutron by the material inside the sample can reduce the energy of the neutron below the discrimination threslold ievel of the plastic scintillation detector, rasulting in a loss of a coincidence count. Both processes, capture and elastic scattering, work to reduce the coincidence rate to a value that is lower thar it should be; the resulting assay will then be low.

The ${ }^{252} \mathrm{Cf}$ source-addition technique ${ }^{7}$ is used to correct the assay for these losses. The attenuation correction is made in the following manner: the coincidence rate of a small spontaneous fission ${ }^{252} \mathrm{C}$ fource $\left(\sim 10^{4} \mathrm{n} / \mathrm{s}\right)$ is determined by placing the source into the Random Driver; the coincidence rate of the source is then determined with the sample in the counter; the ratio of the coincidence rate obtained with the simple in the counter to that obtained without the sample gives an estimate of the decrease in the response due to the material in the sample. The attenuation is a function of both the density and the type of material in the sample. A heavy sample contairing a matrix of $8 \mathrm{~kg}$ of iron, for example, exhibits a coincidence rate that is about $20 \%$ lower per gram of ${ }^{235} \mathrm{U}$ than does a lightweight sample, ${ }^{8}$ The ${ }^{252} \mathrm{Cf}$ source is placed on the outside of the sample about $100 \mathrm{~mm}$ above the rotating turntable on which the sample sits (the source rotates with the sample). Measurements show that the attenuation correction will be approximately the same no matter whether the ${ }^{252} \mathrm{Cf}$ source is located on the outer surface of the sample, or whether it is loc ated anywhere inside the sample; i. e., :he measul ed response is nearly independent of the position of he fission location within the sample.

The ${ }^{252} \mathrm{C}$ f source-addition technique also automatically corrects the assay fo: long-term changes in the response caused by instrumentation drifts. A response increase of $10 \%$, for example, perhaps caused by a severe temperature change or by a shift in photomultiplier tube voltage, will prcduce the same increase in response for both the sample and the ${ }^{252} \mathrm{Cf}$ spontaneous-fission source. The normalization process thus removes the effects of this response change. It is necessary, of course, to periodically correct the normalization factors because of the decay of the ${ }^{252} \mathrm{Cf}$ source $1960 \cdot \mathrm{d}$ halflife).

\section{ASSAY INSTRUMENT}

The completed assay instrument is shown in Fig. 3. The turntable on which the sample is placed is located in the center of the counter between the two fast-plastic scintillation detectors. The fission chamber (Reuter-Stokes model RS-P6$1608-110$ ) is located to the left of the turntable. In practice, two neutron sources, seen at the rear of the counter, are used instead of a single source. A uniform vertical response from the sample $( \pm 3 \%)$ is obtained with the two sources (each 50.8-mmo. d. by 140-mm-long) spaced $250 \mathrm{~mm}$ between centers (see Appendi: A). A narrower spacing between these sources results in a peaking of the

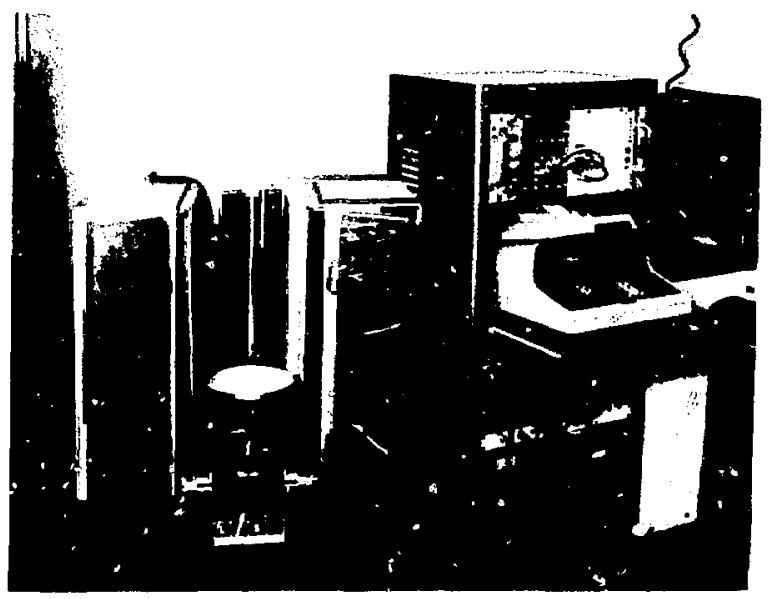

Fig. 3. Completed Randomi Driver assay system for assay of 10-liter containers. 
response at the centur of the sample, whereas a wider spacing results in a peaking of the response at the ends of the sample. The proper spacing for the flat resporse was determined experimentally.

The fast-plastic scintillation detectors are 50.8-mm-thick, 254-mm-wide, and 610-mm-long. The inner face of each detector is covered with a 25.4-mm-thick layer of lead and a 6.35-mm-thick layer of BORAL. The purpose of the lead is to reduce assay nonlinearities in dense samples by preventing the low-energy fission gamma rays, which suffer severe attenuation in these samples, from reaching the detectors. The BORAL keeps the neutron spectrum inside the assay system at high energy by absorbing neutrons that are thermalized in the plastic scintillation detectors and preventing them from reaching the sample.

The two doors, which are shown open in Fig. 3, make it easy for the operator to put the sample into the counter. These doors, which are closed during the assay, are covered with I3ORAL, to eliminate thermalized room-return neutrons.

The coincidence logic circuil is shown in

Fig. 4. Signals from the two photomultiplier tubes (RC A 8575) on each plastic scintillation detector are

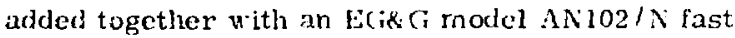
mixer. These signals are then converted to it andard fast-logic pulses ( $40-n s-w i d e)$ by an $E$ C i C model T $121 / \mathrm{N}$ quad discriminator. The coirsidence logic consists of (l) a prompt channel that records both the coincidences from the induced fissions and the accidental (chance) coincidences produced by the landom source and the sample itself, and (2) a delayed-coincidence channel that records only the accidental coincidences. The differetice between the count rates in these two channels is the inducedcoincidence rate, which is proportional to the $2: 35$; content of the sample. The delay for the accidentalcoincidence channel is produced by a delay line made of $30.5 \mathrm{~m}$ of $50-\ldots$ coaxial cable (nc:293). The

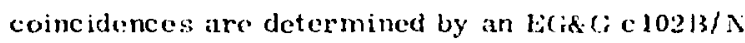
dual-overlap coineidence unit, The coincidence-

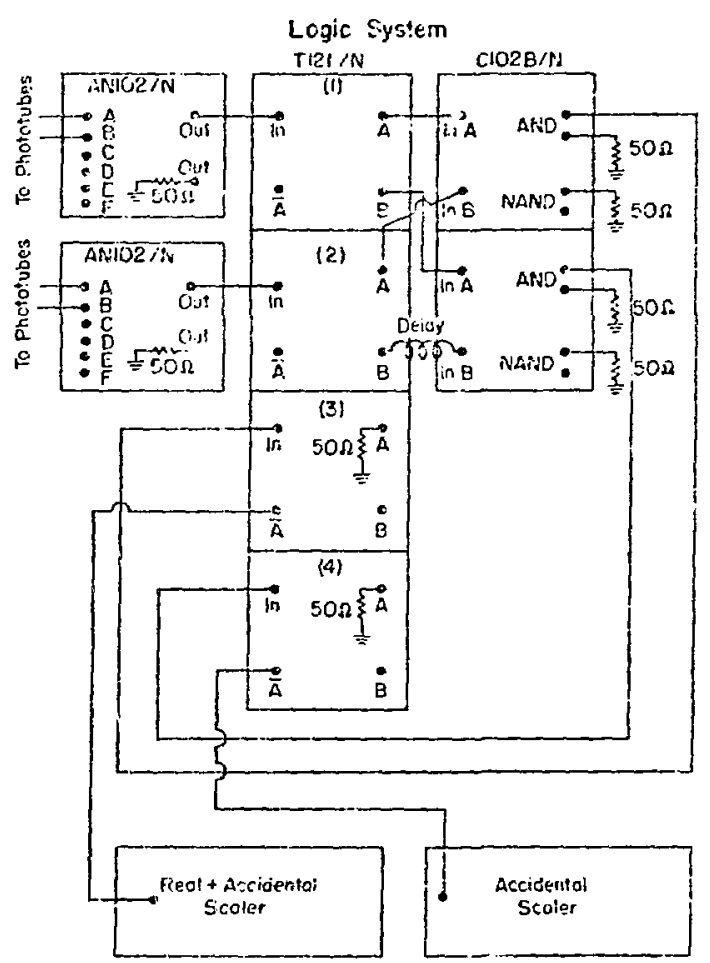

Rote: Ail coties must be $50 \Omega$

Fig. 4. Coincidence Iogic system for the Random Driver.

resolving time is determined by the cutput pulse widths of the discriminators. The outputs of the coincidence units are shaped by the remaining two discriminators in the T121/N module for proper acceptanes by the reunelec model TC $546 P$ scalers.

The calculations for the assay are done automatically by a llewlett-Jackard 9100h programmable calculator that is interfaced to the scalers. The sequence for the assay of a sample is

1. the sumple is weighted and its weight is entered into the calculator,

2. the induced response is determined in a $200-5$ count,

3. the 252 ('i source is then placed into itic counter at the side of the sample,

4. the response of the 252 ( $f$ source is determined in a second 200-s count, and 
5. the assay is automatically calculated; the value is presented on the display, and it is printed on a paper tape for a permanent record.

The equations used fo: the calculations are presented in Appendix $B$.

\section{CALIBRATION}

Four standards, which simulate the Roversamples, were used to calibrate the instrument. These standards, which contain 500, 1000, 2000, and $4000 \mathrm{~g}$ of enriched uranium in "hospital cans," span the mass range of the samples to be assayed. They are composed of a mixture of $93 \%$ enriched $\mathrm{U}_{3} \mathrm{O}_{8}$ powder and graphite flour, filled to within $\sim 25 \mathrm{~mm}$ of the top of the containers.

The calibration curve that was generated using these standards, and using two neutron sources ( $5 \times 10^{6} \mathrm{n} / \mathrm{s}$ each) spaced $250 \mathrm{~mm}$ apart, is shown in Fig. 5, Background, fission chamber, and ${ }^{252} \mathrm{Cf}$ source-addition corrections are included in this calibration curve (see Appendix B).

The nonlinearity in the calibration curve indicates that the assay, unfortunately, is a function of not only the amount of uranium in the sample. but it is also a function of the density of the uranium

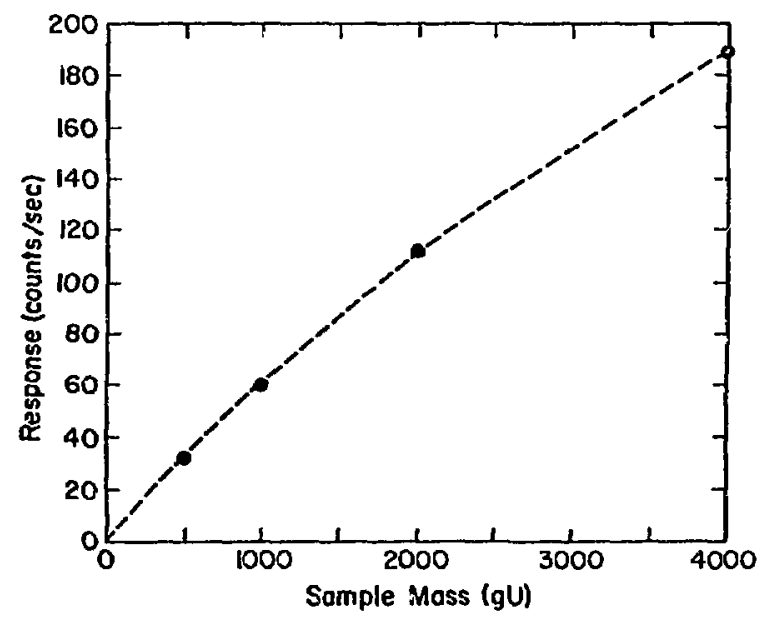

Fig. 5. Calibration curve for 10-1iter containers filled with a mixture of $93 \%$ enriched $\mathrm{U}_{3} \mathrm{O}_{8}$ and graphite nour. in the sample. The response per gram of uranium in the $4000-g$ standard, for example, is $\sim 80 \%$ of the response per gram of the $1000-\mathrm{g}$ standard. This problem can be overcome by filling the samples to be assayed to the same fill height as that of the standards; i. e., the samples will all hav $z$ the same volume. If a sample is reasonably homogeneous, then its mass and its density are proportional, with the proportionality constant being the fixed volume. The calibration curve is then unique and the nonlinearity causes no problem. Accurate assays thus require constant fill heights.

The calibration curve of Fig. 5 is fitted, by the least-squares technique, to an equation of the form 9

$$
\mathbf{R}=\mathbf{A}\left(\mathbf{1}-\mathrm{e}^{\mathrm{BU}}\right) \text {, }
$$

where $R$ is the corrected coincidence rate (see Appendix $B$ ) of the sample containing $U$ grams of $93 \%$ enriched uranium. $A$ and $B$ are calibration constants determined by the least-squares fit. Inversion of this equation to

$$
U=\frac{1}{B} \ln \left(1 .-\frac{R}{A}\right)
$$

gives the assay in grams of uranium.

\section{ASSAY PRECISTON \\ The precision of the assay can te obtained} by propagating the uncertainties associated with the count rates through all of the equations. It is easier, however, to obtain estimates of the precision by making repeated measurements on the standards. Table I gives the results of such measurements; these values can be used as estimates of the precision of measurements of actual samples.

Since the nrecision of the assay of samples with a uranium content of less than $500 \mathrm{~g}$ is goveried almost totally by the background rates, an estimated standard deviation of $\sim 20 \mathrm{~g}$ must be 
TABLE I

\section{ESTIMATED STANDARD DEVIATION OF ROVER SAMPLES}

Sample

$500 \mathrm{~g}$

$1000 \mathrm{~g}$

$2000 \mathrm{~g}$

$4000 \mathrm{~g}$
Standard Deviation

$60 \mathrm{~g}$

$110 \mathrm{~g}$

$290 \mathrm{~g}$ applied to all such samples. The practical minimum sensitivity of the instrument for assay of samples in 10-liter "hospital cans," using a 200-s assay time, is therefore $\sim 50 \mathrm{~g}$ of uranium. A lower sensitivity can be obtained, if necessary, by increasing the assay time. An 800-s assay, for example, will give a sensitivity of $\sim 25 \mathrm{~g}$ of uranium.

Because the actual content of a sample is generally not known accurately, it is difficult to make quantitative evaluations of the accuracy of the Random Driver. However, comparisons have been made between assays made with this instr' $\lrcorner$ ment and assays made with the delayed-neutron technique. The agreement between the two techniques, as shown in Fig. 6, is quite good.

Further evaluations of the performance of the instrument will be made as more information is obtained about sample content from the uranium recovery operations. The major problem encountered in comparing the assay values with the recovery values is that recovery samples are generally

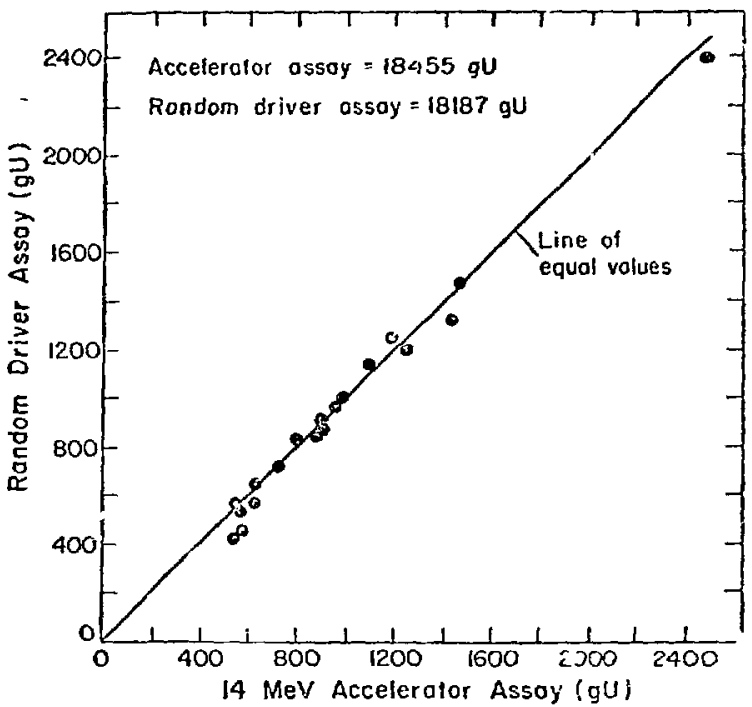

Fig. 6. Comparison of assays of the Ranciom Driver with assays made by the deiaye 1 -neutron technique.

"batched" together into large groups for processing; thus, the individual sample identity is lost and individual comparisons cannot be made.

\section{CRITICALITY PROCEDURES}

The criticality procedures followed in assaying the Rover scrap material are given in Appendix $C$. These procedures apply only to this Random Driver and this particular application. New procedures must be developed for different assay situations.

\section{APPENDD $A$}

\section{VERTICAL RESPONSE OF SYSTEM}

Early Random Drivers ${ }^{10}$ used a source that moved up and down to nelp flatten the vertical response of the sample. The response, however, was still higher at the center of the sample than it was at the ends. The response at the center of the sample can be reduced, and at the same time the response at the ends increased, if two sources are used. These sources are spaced such that the response at the center and at the ends of the sample are the same. This technique works well for source spacings of up to $250 \mathrm{~mm}$. When spacings are larger than this, the response in the center of the 
sample is lower than at the ends. Tall samples that require large source spacings may require an additional source ("shim source") at the center of the counter to boost the response of the center of the sample. Figure A-1 shows the vertical response of the Random Driver for three source configurations: (1) a source fixed in the center of the counter, (2) a source moving up and down at a uniform speed with amplitude of $\neq 125 \mathrm{~mm}$, and (3) two sourses with centers spaced $250 \mathrm{~mm}$ apart. These measurements were made by placing the center of a $70-\mathrm{mm}-$ thick layer of material (250-mm-0.d.) at various distances from the center of the counter. Each data point was measured to a precision $(1 \sigma)$ of $\sim 1 \%$.

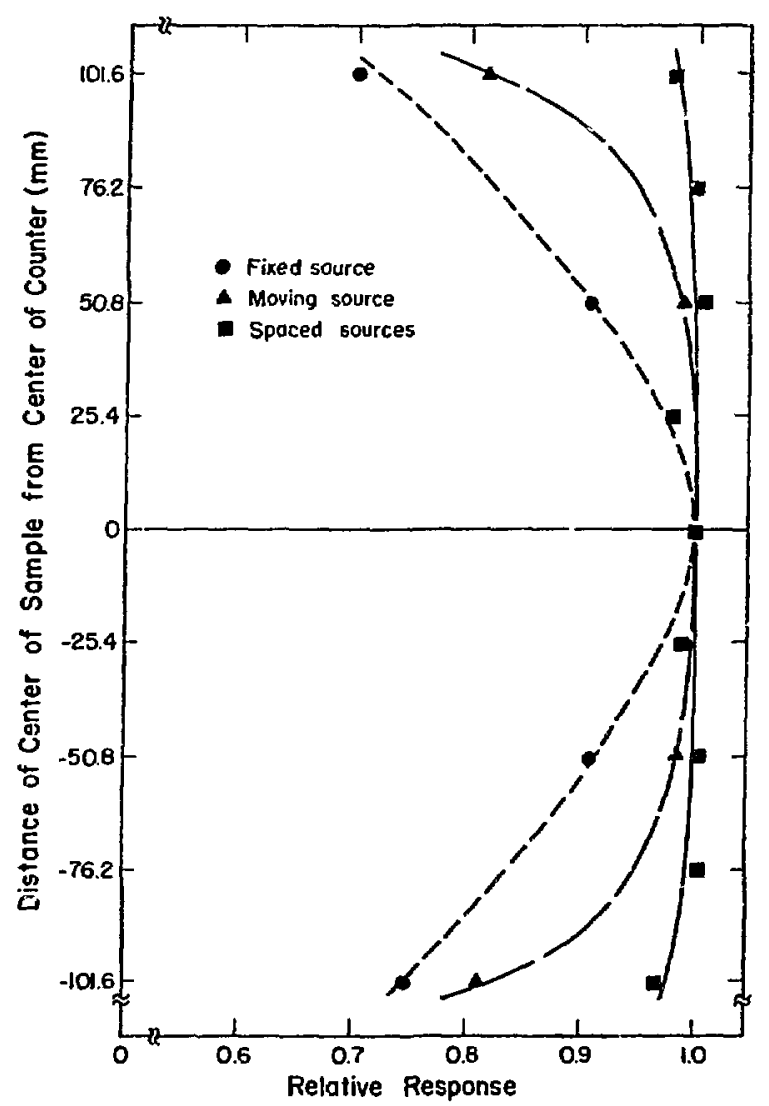

Fig. A-1. Vertical response profiles of the Random Driver for different source conditions. 


\section{CALCULATIONS}

A

Eackground Correction

The background coincidence rate $\vec{B}$ is determined from the weight of the sample using Fig. 2. B. Induced-Coincidence Rate

The induced-coincidence rate when the sample is in the counter is given by

$$
\dot{I}=\left(\dot{R}_{1}-\dot{R}_{1}-\dot{B}\right),
$$

where $\dot{R}_{1}$ is tne count rate of the prompt-coincidence scaler, and $\dot{R}_{1}^{\prime}$ is the count rate of the delayedco:ncidence scaler.

\section{Fission Chamber Correction}

The fission chamber response when there is no sample in the counter is given by $i_{0}$ and the response with the sample is $\dot{A}_{\mathrm{s}^{*}}$ The fission charnber correction factor $\mathrm{G}$ is then

$$
G=\frac{\dot{A}_{o}}{\dot{A}_{S}} .
$$

$\underline{\text { D. }}$

\section{Attenuation Correction}

The ${ }^{252} \mathrm{Cf}$ source-addition correction is made in the following manner.

Let $\dot{R}_{2}$ and $\dot{R}_{2}$ be the coincidence rates of the prompt and the delayed scalers, respectively, when the ${ }^{252} \mathrm{Cf}$ source is in the counter without the sample.
The coincidence rate of the ${ }^{252} \mathrm{Cf}$ source $\dot{C}_{0}$ is then $\dot{\mathrm{C}}_{\mathrm{o}}=\left(\dot{\mathrm{R}}_{2}-\dot{\mathrm{R}}_{2}^{\prime}-\mathrm{B}\right)$

Let $\dot{R}_{3}$ and $\dot{R}_{3}$ be the coincidence rate of the prompt and delayed scalers, respectively, when the ${ }^{252} \mathrm{Cf}$ source is in the counter with the sample. The coincidence rate of the ${ }^{252} \mathrm{Cf}$ source in the presence of the sample $\dot{C}_{s}$ is then

$$
\begin{aligned}
& \dot{C}_{s}=\left(\dot{R}_{3}-\dot{R}_{3}^{\prime}-\dot{B}-\dot{I}\right) . \\
& \text { The }{ }^{252} \text { Cf source-addirion correction fac- }
\end{aligned}
$$
tor $\mathrm{F}$ is then given by

$$
F=\frac{\dot{C}_{o}}{\dot{C}_{s}}
$$

\section{E. Corlected Sample Resporse}

The sample coincidence rate with background correction, fission chamber correction, and attenuation correction is then

$$
\boldsymbol{R}=\mathbf{G} \cdot \mathbf{F} \cdot \dot{\mathbf{I}}
$$

The final assay is then determined from Eq. (2). 


\section{APPENDLX $C$}

\section{CRITICAIITY PROCEDURES *}

The following procedure shall apply for the assay of uranium ( $593.5 \%{ }^{235} U$ ) in the Random Driver.

Any container having gross weight less than $3 \mathrm{C}$ ib ( $13.6 \mathrm{~kg}$ ) and volume less than 20 liters may be placed in the Random Driver for assay, providing the air gap between the sides or the container and the Random Driver is at least $2 \mathrm{~cm}$.

These limits are based on a series of DTF7 1 calculations (using Hansen-Roach 16-group cross sections) in which the Random Driver was modeled as a series of spherical shells (Boral, Fe, $\mathrm{H}_{2} \mathrm{O}$ ) surrounding a spherical volume containing $\mathrm{U}$ (93. $5 \%{ }^{235}(\mathrm{U})+\mathrm{H}_{2}$ ). A 2-cm gap was maintained between the $\mathrm{U}+\mathrm{H}_{2} \mathrm{O}$ mixture and the innermost shell of the reflector. The calculations indicate the minimum total mass of a critical mixture of $U$ $\left(93.5 \%{ }^{235} \mathrm{U}\right)$ and water in this system to be $\sim 14.6$ kg.

The 30-1b ( $213,6-\mathrm{kg}$ ) limit is considered conservative for the following reasons.

1. Calculations by Stratton ${ }^{11}$ for unreflected and $\mathrm{H}_{2} \mathrm{O}$ fully reflected $\mathrm{U}(93.5 \%)+\mathrm{H}_{2} \mathrm{O}+$ C mixtures indicate that $\mathrm{U}+\mathrm{H}_{2} \mathrm{O}$ (no C) results in the lowest critical mass of ${ }^{235} U$ and the lowest gross - aight for any given volume in the range that will fit into the Random Driver.

2. The Random Driver has hydrogenous reflectors only in two sides; the front and rear are reflected only by $25.4 \mathrm{~mm}$ of steel, and the top is completely unreflected. Thus the spherical geometry used in the calculations should be quite conservative.

\footnotetext{
This appendix written by D, B. Smith, A-1 (J,ASL).
}

3. The gross weight limit set above is $7 \%$ below the minimum total mass indicated by the calculations and includes the weight of the empty container.

\section{REFERENCES}

1. J. H. Menzel, A, C. Dumrose, and R. H. Augustson, I.OS Alamos Scientific Laboratory report LA-4794-MS (197 I), p. 1 .

2. J. L. Parker, T. D. Reilly, J, E. Foley, F. B. Walton, and L. V. East, "Passive Assay--Innovations and Applications," Proceedings of the Twelfth Annual Meeting of the Institute of Nuclear Materials Management, June 29-.July 1, 197 1, Vol. II, pp. 5 14-547.

3. J. E. Foley, U. S. Patent $3,736,429$, issued May 29, 1973.

4. J. E. Foley, "Application of the Random Source Interrogation System (Random Driver) at the Oak Ridge Y-12 Plant--Preliminary Results," Los Alamos Scientific Laboratory report LA-5078-MS (1972).

5. Ibid., p. 4.

6. Los Alamos Scientific Laboratory report LA-4029-MS (1968), p. 5 .

7. H. O. Menlove, Los Alamos Scientific Laboratory report LA-4457-MS (1970). p. 29.

8. J. E. Foley, Los Alamos Scientific Laboratory report LA-5431-PR (1973), p. 5.

9. R. A. Forster, D. B. Smith, and H. O. Menleve. "Error Analysis of a ${ }^{252} \mathrm{Cf}$ FuelRod-Assay Systcrn," LA-53 17 (1974), p. 10.

10. J. E. Foley, Los Alamos Scientific Laboratory report LA-488:-PR (1973), p. $\%$

11. W. R. Stratton, "Criticality Data and Factors Affecting Criticality of Single Homogeneous Unit 3, " Los Alamos Scientific Laboratory report LA-36 12 (1967), p. 16. 